\title{
预防中风伤及大脑的突破性技术 血液替代疗法
}

林佑

香港大学中医药学院

如果你觉得半身肌肉无力, 而且说话逐渐变得 含糊不清, 这可能是中风的迹象, 得赶紧去急诊室 就医检查。

根据美国疾病控制及预防中心的统计显示, 每 年超过 79.5 万美国人经历中风, 14 万人死于中风。 这是一种突发性的疾病, 一但发作病情十分紧急, 如果未能及早救治, 很可能会导致半身不遂或变成 植物人，甚至危及生命。

目前针对脑中风的药物十分有限, 比较有代表 性的是, 早前 FDA 批准的缺血性中风药物——组织 纤溶酶原激活剂 (tPA)。该药物是通过激活纤维溶 解酶, 把堵塞在通往大脑动脉的血栓溶解, 回复心 脑血管的正常血液循环。问题是，tPA 必须在中风后 $3 \mathrm{~h}$ 内给药，否则没有效果。而且药物对脑损伤沒有 保护作用。

医学家必须寻找用途更广、效果更全面的治疗
中风的方法。

\section{研究报告}

近年來, 西弗吉尼亚大学神经科学家进行一项 中风小鼠研究实验, 研究小组发现血液替代疗法可 以避免小鼠脑缺血损伤。研究成果已发表在《自然 通讯》期刊。实验团队由神经科学系研究助理教授 任雪芳 (Xuefang 'Sophie' Ren) 领导。

\section{血液替代疗法}

血液替代疗法, 就是把健康小鼠的部分血液注 射到中风小鼠体内, 并从病鼠体内抽走相同容量的血. 液, 这种新旧血液交换的方式, 可以让病情得显著

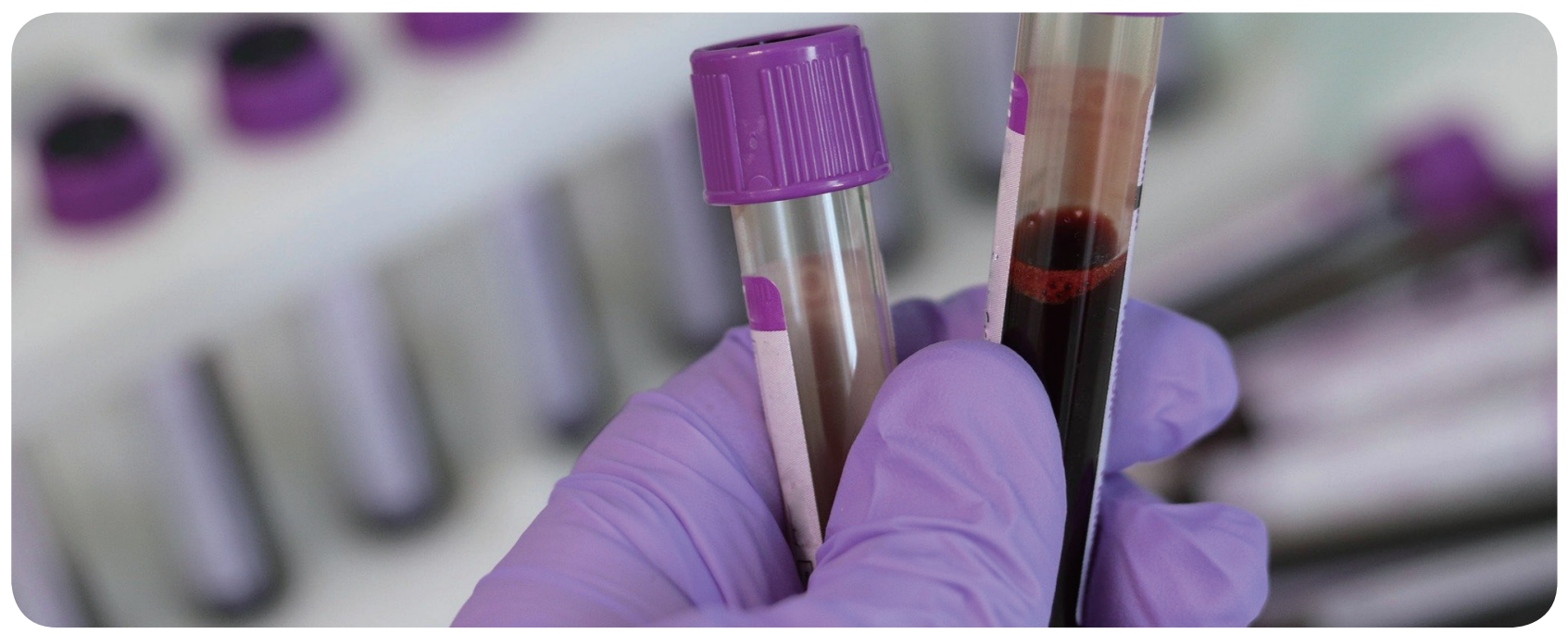


改善, 对脑神经的损伤风险大幅降低。

\section{血液替代疗法原理}

我们知道, 中风后血液会发生变化, 在这过程 会触发免疫系统反应, 免疫细胞会进入大脑, 试图 清除造成脑损伤的有害物质, 但由于过多免疫细胞 积聚脑部, 容易引起炎症。更糟糕的是, 其中一种 的免疫细胞 (中性粒细胞) 会提高 MMP-9 酶水平, 这种酶可以导致血脑屏障渗漏和脑组织退化, 令脑 部严重受损。

血液替代疗法可以清除中风后血液残伤物质, 包括相关的免疫细胞、炎症因子及酶类，有效保护 脑神经及脑组织。

\section{血液替代疗法优点}

1）作用时间更长: 对于中风病情长达 $7 \mathrm{~h}$ 以上
的动物而言仍然有积极的作用。

2) 操作简单可行: 只要换掉病患小鼠 20\%的血. 液, 足以大幅降低脑部损伤。这样的血量, 相对成 年人体内约 1.5 加仑的血液來看, 是可行可操作的。

\section{结论}

研究人员指出, 近年以血液为基础的治疗方法 对抗衰老和神经退行性疾病进行广泛研究, 并取得 可喜的成绩。

他们首次利用血液替代疗法用于中风动物身上, 取得明显改善效果。下一步需要进行临床实验证明 这个研究结果是正确的。

\section{参考文献}

[1] Medical Xpress. New blood, new hope: Transfusions protect the brain from stroke damage. Aug 25, 2020. https:// medicalxpress.com/news/2020-08-blood-transfusionsbrain.html.

\section{热烈祝贺《医学研究与发表》被多所985、211高校图书馆纳入馆藏}

《医学研究与发表》(Medical Research \& Publication, 以下简称 MRP) 创办于 2015 年 6 月。本刊是由《医学研究与 发表》编辑部主办、华誉出版社出版、美捷登生物科技有限公司及武汉华易研生物科技有限公司联合赞助的公益性学术 季刊。MRP 期刊 ISSN 号为:

\section{Medical Research \& Publication (Print) ISSN : 2475-7500 \\ Medical Research \& Publication (Online) ISSN : 2475-7543}

本刊办刊宗旨为深入探讨如何有效地进行医学研究和发表, 快速传播最新的医学研究动态、及时分享医学研究和 论文写作投稿的知识和经验, 本着 “做真实研究, 出创新成果, 发高质文章, 办优秀期刊” 的理念, 为广大医学研究者 提供学习、交流和探索的平台, 共同促进医学研究的发展。

MRP 内容兼具学术性和实用性。纸质版 MRP 排版雅致, 打印精美。目前, 国内已有多所 985、211 高校图书馆订 阅纸质版 MRP，并将其纳入馆藏。部分高校图书馆名单如下 (按汉语拼音排列 ) :

\begin{tabular}{|c|c|c|c|}
\hline 长沙医学院图书馆 & $\begin{array}{l}\text { 重庆医科大学附属 } \\
\text { 第二医院图书馆 }\end{array}$ & 东北师范大学图书馆 & 复旦大学图书馆 \\
\hline 广州医科大学图书馆 & 华南理工大学图书馆 & 华中科技大学图书馆 & $\begin{array}{l}\text { 华中科技大学同济医学 } \\
\text { 院附属同济医院图书馆 }\end{array}$ \\
\hline $\begin{array}{l}\text { 吉林大学第一医院图书馆 } \\
\text { 天津中医药大学图书馆 }\end{array}$ & $\begin{array}{l}\text { 暨南大学图书馆 } \\
\text { 武汉大学图书馆 }\end{array}$ & $\begin{array}{l}\text { 江南大学图书馆 } \\
\text { 西南交通大学图书馆 }\end{array}$ & 南京大学图书馆 \\
\hline
\end{tabular}

